\title{
Pontos marcados na superfície da pele representam os processos espinhosos quando a postura é modificada?
}

\author{
Can skin markers represent spinous process when the posture is changed?
}

Tássia Silveira Furlanetto ${ }^{1}$, Fabiana de Oliveira Chaise ${ }^{2}$, Cláudia Tarragô Candotti ${ }^{3}$, Tatiana Comerlato ${ }^{4}$, André Fernando da Rocha ${ }^{5}$, Jefferson Fagundes Loss ${ }^{6}$

Estudo desenvolvido na ESEF - Escola Superior de Educação Física da Universidade Federal do Rio Grande do Sul (UFRGS) - Porto Alegre (RS), Brasil.

${ }^{1}$ Mestranda bolsista Capes de Neurociências da Universidade Federal do Rio Grande do Sul (UFRGS) - Porto Alegre (RS), Brasil.

${ }^{2}$ Graduanda em Fisioterapia da Universidade do Vale do Rio dos Sinos (UNISINOS) -São Leopoldo (RS), Brasil.

${ }^{3}$ Doutora; Docente do Curso de Fisioterapia da UFRGS - Porto Alegre (RS), Brasil.

${ }^{4}$ Mestre em Ciências do Movimento Humano da UFRGS - Porto Alegre (RS), Brasil.

${ }^{5}$ Fisioterapeuta; Mestrando em Tecnologias de Materiais e Processos Industriais da Universidade FEEVALE - Novo Hamburgo (RS), Brasil.

${ }^{6}$ Doutor; Docente do Curso de Educação Física da UFRGS Porto Ảlegre (RS), Brasil.

ENDEREÇO PARA CORRESPONDÊNCIA:

Tássia Silveira Furlanetto - Rua Bento Alves, 1.501 - apto. 204 Bairro Rio Branco - CEP: 93032060 - São Leopoldo (RS), Brasil - E-mail: tassiasf@gmail.com.

APRESENTACÃO

abr. 2010

ACEITO PARA PUBLICAÇÃO jan. 2011

FONTE DE FINANCIAMENTO: CAPES

CONFLITO DE INTERESSES: nada a declarar

Parecer de aprovação no Comitê de Ética n ${ }^{\circ} 2006660$.
RESUMO: O objetivo do estudo foi verificar radiograficamente se a posição dos marcadores de superfície, identificados por palpação em ortostase, é alterada com a modificação da postura para o decúbito lateral. Dez indivíduos foram: palpados e marcados com adesivos de superfície, os processos espinhosos das vértebras C7, T2, T4, T6, T8, T10, T12, L2, L4 e S2; submetidos a um exame radiológico da coluna vertebral na posição ortostática e em decúbito lateral direito para verificar se o marcador de superfície colocado sobre o processo espinhoso estava condizente com o processo espinhoso da imagem radiográfica. Uma ANOVA two-way mostrou diferenças significativas para o deslocamento do marcador de superfície entre as posições para as vértebras C7, T2, T4, T6, T8, T10, L2 e S2 ( $\mathrm{p}<0,001)$. Nas vértebras T12 e L4 não foram encontradas diferenças significativas. Os resultados indicam que a localização do marcador de superfície não foi condizente com o processo espinhoso identificado no exame radiológico, entre as posições. O movimento da pele pode ter deslocado o marcador de superfície, quando o indivíduo modificou sua posição da posição ortostática para decúbito lateral direito.

Descritores: palpação; postura; radiografia.

ABSTRACT: The objective was to radiographically verify if the position of skin markers, identified by palpation in orthostasis posture, changes when the subject position shifts to lying posture. Ten subjects underwent two X-ray tests, first, in orthostasis and right lying posture. Ten subjects were palpated and marked with skin markers in the orthostasis posture, in the spinous process of the vertebrae C7, T2, T4, T6, T8, T10, T12, L2, L4 and S2 . After palpation, subjects underwent X-ray exams in orthostasis and lying postures to verify if the surface marker matched the spinous process shown on X-ray. A two-way ANOVA indicated significant differences for the displacement of the skin markers, for the C7, T2, T4, T6, T8, T10, L2 and S2 spinous processes $(p<0.001)$. The T12 and L4 spinous processes did not indicate significant differences. The skin markers locations were not consistent with the spinous processes identified in X-rays for both positions. The skin movement may have displaced the skin markers when the subject changed from orthostasis to lying posture.

KEYWORDS: palpation; posture; radiography. 


\section{INTRODUCÃO}

Tradicionalmente, para analisar os desvios posturais, os fisioterapeutas utilizam a avaliação postural, a qual permite identificar as alterações de cada segmento corporal específico e as modificações nas estruturas globais do indivíduo ${ }^{1}$. Para isso, o profissional realiza a inspeção visual ${ }^{1}$ e, conforme as alterações observadas, tem condições de planejar e executar um tratamento corretivo e preventivo por meio de técnicas que permitam uma melhora da condição postural e funcional do indivíduo. No entanto, outras maneiras existem de avaliar a postura global, seja por meio de fotografias digitais ${ }^{2-5}$ ou instrumentos de medida ${ }^{6-15}$. As fotografias digitais, atualmente vinculadas a softwares, permitem maior riqueza de observações e objetividade quanto à identificação das modificações funcionais e estruturais do indivíduo ${ }^{16-21}$

Para a utilização desse recurso, necessita-se identificar e demarcar alguns pontos anatômicos de referência, utilizando marcadores de superfície sobre a pele do indivíduo ${ }^{2-6,22}$. Essa marcação ocorre por meio do método palpatório das proeminências ósseas, por isso, uma correta palpação está intimamente relacionada ao sucesso na avaliação ${ }^{5,23-25}$. A palpação dos processos espinhosos (PE) é a etapa mais importante e condicionante para que a avaliação seja válida e consistente, erros nessa etapa determinam falsos possíveis desvios e alterações da coluna vertebral (CV) nos planos sagital e frontal. Esta etapa executada corretamente permite que o profissional possa seguir executando a metodologia de avaliação postural com mais confiança ${ }^{26}$.

Avaliações posturais por esse método também são realizadas em outras situações, como na análise da manutenção das curvaturas da CV durante a corrida em esteira ${ }^{27}$, ou análise da acomodação da CV em decúbito lateral, partindo da posição de ortostase ${ }^{28}$. A qualidade das avaliações nestas situações está intimamente ligada à manutenção da representatividade das proeminências ósseas pelos pontos marcados sobre a superfície da pele. Neste sentido, parece pertinente o seguinte questionamento: mudanças de postura (por exemplo, da postura ortostática para decúbito) acarretam em deslocamentos dos marcadores de superfície posicionados sobre a CV na posição ortostática, levando a análises errôneas? Se por um lado a pergunta é pertinente, a resposta a esta questão não é tão simples de ser obtida. Para situações como as anteriores ${ }^{27,28}$, há uma grande dificuldade de comprovar que os pontos

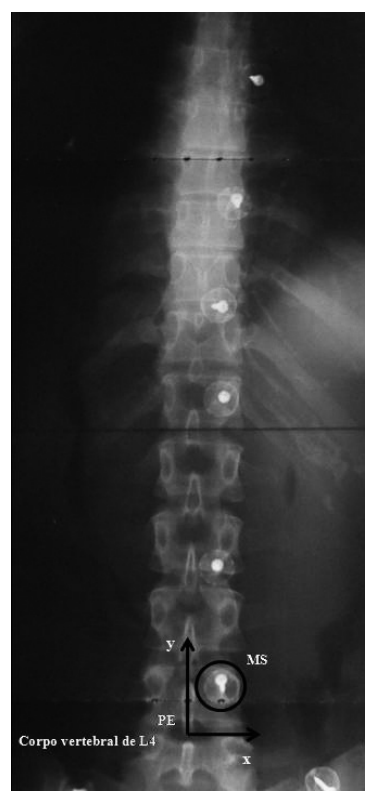

Figura 1. Imagem radiológica da coluna vertebral no plano frontal, em decúbito lateral direito. As setas indicam sistema de coordenadas cartesianas $(x, y)$, em que o centro do Processo Espinhoso serve como origem

marcados na pele realmente representam os processos espinhosos, sendo necessária uma confirmação obtida por exames de raios $X$. Sendo assim, o objetivo deste estudo foi verificar radiograficamente se a posição de marcadores de superfície, identificados por palpação em ortostase, é alterada com a modificação da postura para decúbito lateral.

METODOLOGIA

A amostra foi constituída por dez indivíduos que apresentavam em média $31,9 \pm 12,3$ anos de idade, 58,4 $49,4 \mathrm{~kg}$ de massa corporal e 1,66 $\pm 0,07 \mathrm{~m}$ de estatura. O critério de inclusão foi possuir requisição para realização do exame de raios $X$ da coluna vertebral no plano frontal. Os critérios de exclusão foram: apresentar seis vértebras lombares, doenças ou deficiências vertebrais, impossibilidade de permanecer em ortostase e contraindicações para a realização dos exames de raios $X$. Os indivíduos assinaram um termo de consentimento livre e esclarecido, autorizando a participação no estudo. O presente estudo foi aprovado no Comitê de Ética da instituição envolvida no estudo, sob o número 2006660.

Os Processos Espinhosos das vértebras C7, T2, T4, T6, T8, T10, T12, L2, L4 e S2 foram identificados, por meio do método palpatório, e demarcados com marcadores de chumbo, estando o indivíduo na posição ortostática (PO). Levando em consideração que o método palpatório é dependente da experiência do avaliador ${ }^{25,29,30}$, os procedimentos de palpação foram realizados por um único fisioterapeuta, com sete anos de experiência na prática clínica em demarcação de pontos anatômicos. Imediatamente após a marcação dos $\mathrm{PE}$, os indivíduos foram submetidos no mesmo dia e local a dois exames de raios $X$ da $C V$, um na $P O$ e outro em decúbito lateral direito (DLD), ambos no plano frontal com incidência posteroanterior. $\mathrm{O}$ primeiro exame de raio $X$ foi realizado na $P O$, no qual o indivíduo foi posicionado em pé de frente para o chassi, com os membros superiores relaxados ao longo do corpo e com o peso corporal distribuído igualmente entre os membros inferiores. Imediatamente após, para realizar o segundo exame de raios $X$, o participante foi instruído 
a deitar-se em DLD em uma maca, de frente para o chassi, com a cabeça apoiada em um travesseiro e com leve flexão das articulações coxo-femoral e joelhos. Após a realização deste exame, por meio de um negatoscópio, verificou-se se o marcador de superfície (MS) colocado sobre o PE, identificado pelo método palpatório, estava condizente com o PE da vértebra avaliada. Para identificar se houve correta palpação dos $\mathrm{PE}$, realizada pelo avaliador, foi adotado um sistema de coordenadas cartesianas $(x, y)^{29}$, em que cada PE serviu como ponto de referência, ou seja, o centro do PE foi considerado a origem $(0,0)$, o eixo y foi orientado na direção crânio-caudal (CC) e o eixo x orientado na direção látero-lateral (LL), como pode ser visto na Figura 1.

Quando houvesse uma correta palpação pelo avaliador, o MS estaria posicionado sobre o $\mathrm{PE}$, ou seja, na posição $(0,0)$ e não haveria nenhum deslocamento em qualquer um dos dois eixos do sistema de coordenadas. Caso a correta palpação não fosse atingida, era, então, utilizada uma régua com precisão de $1 \mathrm{~mm}$ diretamente sobre a imagem radiográfica, para quantificar a posição do $\mathrm{MS}$ em relação ao $\mathrm{PE}$, podendo este apresentar um deslocamento CC e/ou LL. Este procedimento foi realizado nas duas posições para cada indivíduo e, em seguida, foram calculadas as médias dos deslocamentos do MS na PO e DLD para todas as vértebras, nos eixos $x$ e $y$.

A análise estatística foi realizada no Software SPSS 13.0. A normalidade dos dados (teste de Shapiro Wilk) e a homogeneidade das variâncias (teste de Levene) foram confirmadas. Para verificar se existia diferença significativa dos deslocamentos de MS em relação ao $P E$, nos eixos $x$ e $y$, entre PO e DLD, foi utilizado ANOVA two-way (postura $X$ eixos). O nível de significância adotado foi de 0,05 .

\section{RESULTADOS}

Os valores médios do deslocamento do MS na PO para a maioria das vértebras apresentaram mínimos deslocamentos (menores que $10 \mathrm{~mm}$ ) tanto no eixo $\mathrm{x}$, quanto no y (Tabela 1), indicando que o MS está praticamente alinhado ao PE
Tabela 1. Médias e desvio padrão (dp) (valores em $\mathrm{mm}$ ) do deslocamento dos marcadores de superfície nas posições ortostáticas e decúbito lateral direito para cada processo espinhoso analisado

\begin{tabular}{ccccccc}
\hline Eixo X & & \multicolumn{5}{c}{ Eixo Y } \\
& Ortostase & Decúbito & & Ortostase & Decúbito & \\
\hline Vértebra & Média \pm dp & Média $\pm d p$ & Valor $p$ & Média $\pm d p$ & Média $\pm d p$ & Valor $p$ \\
S2 & $0,0 \pm 3,8$ & $15,3 \pm 9,8$ & $\mathrm{p}<0,01$ & $-1,8 \pm 8,0$ & $9,8 \pm 21,6$ & $\mathrm{p}=0,15$ \\
L4 & $0,0 \pm 3,9$ & $10,1 \pm 11,8$ & $\mathrm{p}=0,29$ & $25,7 \pm 16,9$ & $28,5 \pm 37,3$ & $\mathrm{p}=0,83$ \\
L2 & $1,9 \pm 4,4$ & $15,8 \pm 8,3$ & $\mathrm{p}<0,01$ & $-1,6 \pm 26,0$ & $24,1 \pm 29,2$ & $\mathrm{p}=0,05$ \\
T12 & $2,7 \pm 3,8$ & $8,5 \pm 10,1$ & $\mathrm{p}=0,10$ & $-3,7 \pm 23,3$ & $8,4 \pm 44,3$ & $\mathrm{p}=0,45$ \\
T10 & $0,0 \pm 4,0$ & $10,0 \pm 4,1$ & $\mathrm{p}<0,01$ & $-12,0 \pm 20,0$ & $7,1 \pm 39,9$ & $\mathrm{p}=0,19$ \\
T8 & $0,0 \pm 4,5$ & $16,9 \pm 6,1$ & $\mathrm{p}<0,01$ & $-10,3 \pm 18,8$ & $12,9 \pm 36,3$ & $\mathrm{p}=0,09$ \\
T6 & $0,0 \pm 3,9$ & $17,6 \pm 4,7$ & $\mathrm{p}<0,01$ & $-1,8 \pm 19,0$ & $22,1 \pm 32,4$ & $\mathrm{p}=0,06$ \\
T4 & $-0,1 \pm 3,8$ & $17,0 \pm 7,3$ & $\mathrm{p}<0,01$ & $8,6 \pm 20,5$ & $36,8 \pm 33,0$ & $\mathrm{p}=0,34$ \\
T2 & $-1,0 \pm 3,7$ & $13,3 \pm 6,3$ & $\mathrm{p}<0,01$ & $12,5 \pm 14,5$ & $53,7 \pm 22,3$ & $\mathrm{p}<0,01$ \\
C7 & $-0,9 \pm 4,1$ & $10,4 \pm 10,1$ & $\mathrm{p}<0,01$ & $15,3 \pm 14,4$ & $58,0 \pm 20,7$ & $\mathrm{p}<0,01$ \\
\hline
\end{tabular}

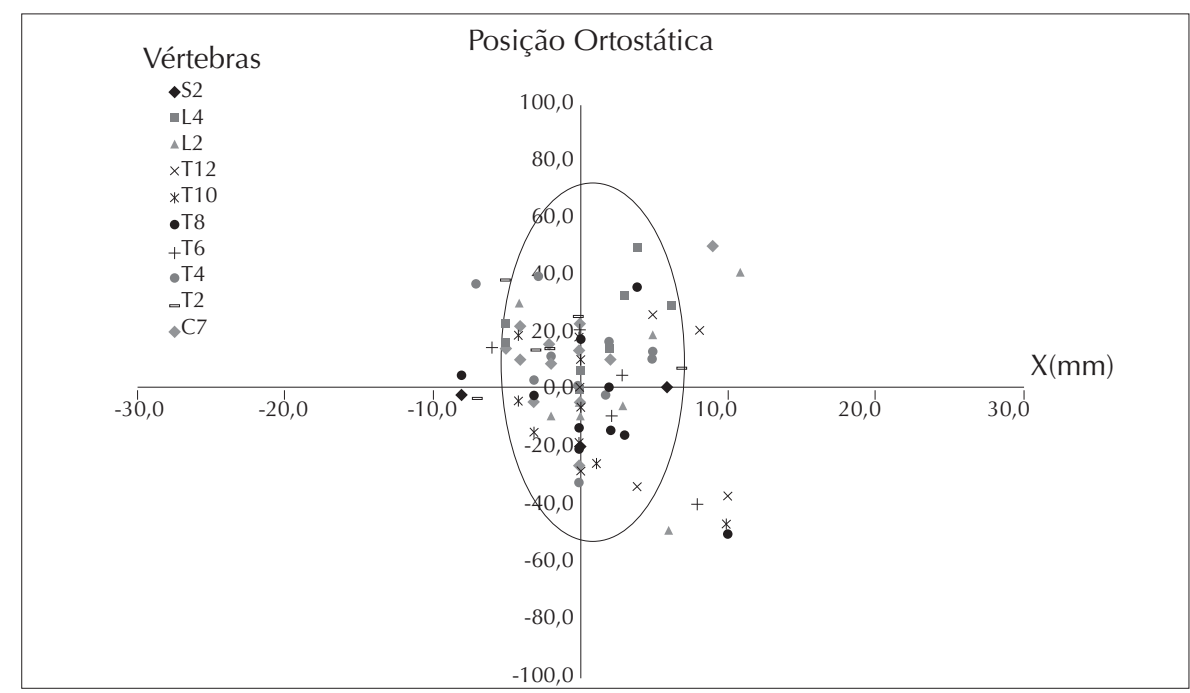

Figura 2. Representação gráfica do deslocamento dos marcadores de superfície nos eixos x e y para as vértebras C7 a S2 para todos os indivíduos na posição ortostática

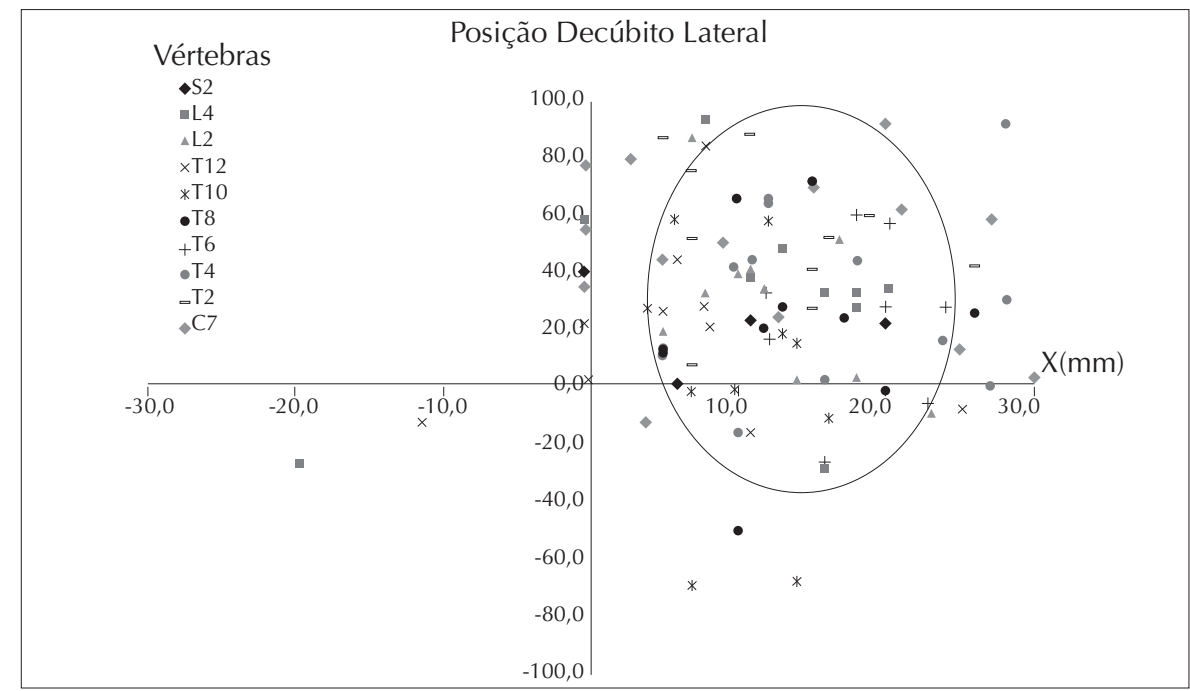

Figura 3. Representação gráfica do deslocamento dos marcadores de superfície nos eixos x e y para as vértebras C7 a S2 a todos os indivíduos na posição decúbito lateral direito 
(Figura 2), com pequenas alterações, tanto CC, quanto LL.

Já na posição DLD, os deslocamentos médios do MS na maioria das vértebras apresentaram valores médios acima de $10 \mathrm{~mm}$ com sinais positivos, tanto no eixo $x$, quanto no y (Tabela 1). Esses resultados indicam que, quando os indivíduos foram instruídos a colocar-se em DLD, houve um deslocamento do MS no sentido para cima e para a direita (Figura $3)$. As exceções foram para as vértebras T10 eT12, as quais apresentaram valores de deslocamento do MS abaixo de 10 $\mathrm{mm}$ em ambos os eixos.

Os resultados da ANOVA ilustrados na Tabela 1 mostraram que o deslocamento do MS entre a PO e DLD apresentou diferenças significativas $(p<0,05)$ entre as posições: nos dois eixos avaliados para as vértebras C7 e T2 esomente no eixo $x$ para as vértebras T4, T6, T8, T10, L2 e S2. Nas vértebras T12 e L4, não foram encontradas diferenças significativas em relação ao deslocamento do MS entre a PO e DLD (Tabela 1).

\section{DISCUSSÃO}

Para avaliação da coluna vertebral, a partir do método palpatório, é necessário que haja exatidão na identificação dos PE. A questão que norteou o presente estudo foi se a posição dos MS, colocados sobre os PE na PO, sofreria alteração com a mudança de postura, e o principal resultado demonstrou que, quando os indivíduos modificaram sua postura, da PO para DLD, ocorreram deslocamentos significativos dos MS na maioria das vértebras avaliadas.

Saad et al. ${ }^{28}$ propuseram um método quantitativo de avaliação da postura em decúbitos lateral e dorsal, baseado em fotografia digital. Para essa avaliação, a identificação das saliências ósseas era realizada uma única vez na $\mathrm{PO}$ e era registrada uma fotografia de cada posição de decúbito (lateral e dorsal). Os resultados de Saad et al. ${ }^{28}$ foram baseados na premissa que as marcações realizadas sobre a superfície da pele na PO manteriam sua relação com os $\mathrm{PE}$, mesmo com o reposicionamento do indivíduo para as posições de decúbito lateral e dorsal. Considerando que os achados do presente estudo demonstraram existir uma alteração significativa na posição do MS sobre a pele, os resultados obtidos por Saad et al. ${ }^{28}$ podem ser questionados.

As diferenças significativas encontradas nos deslocamentos do MS em relação ao $\mathrm{PE}$, entre as posições avaliadas para a maioria das vértebras que compõem a convexidade torácica, podem estar relacionadas ao movimento dos tecidos moles $^{32,33}$, no momento em que o participante modifica a PO para DLD para a realização do segundo exame radiológico. Um dos tecidos moles é a pele, cuja propriedade viscoelástica, bem como a espessura, pode ser uma das responsáveis pelo deslocamento do MS, pois quando os indivíduos foram instruídos a deitar-se em decúbito lateral, os MS deslocaram-se para o lado direito e para cima, acompanhando o contorno da região ${ }^{34}$.

Embora o movimento entre vértebras torácicas seja restrito devido à proteção aos órgãos internos, a região é composta também pelas costelas e pelo esterno ${ }^{35,36}$, que juntos formam o arcabouço torácico, cuja composição envolve uma gama de músculos para a produção do movimento de complacência torácica durante a respiração ${ }^{37}$. Desse modo, especula-se que a elasticidade da pele e da caixa torácica juntamente com a troca de posição do participante possa ter aumentado a mobilidade da região, admitindo que os tecidos moles acompanhem a superfície durante a troca de posição, acarretando no deslocamento do MS. Esses pressupostos levam a inferir que a palpação dos PE deva acontecer na posição em que se deseja avaliar o indivíduo, com o intuito de evitar erros no procedimento avaliativo, bem como a interferência dos tecidos moles.

Cabe ressaltar que, mesmo havendo o controle das curvaturas fisiológicas da CV durante a troca de posição, pode ter ocorrido um pequeno movimento de flexão da $C V$, especialmente na região torácica ${ }^{1}$, ocorrendo um deslizamento superior e ligeiramente anterior das superfícies articulares, além da tração dos ligamentos inter e supraespinhal, levando assim ao movimento dos PE nesta região ${ }^{36}$. Moga et al. ${ }^{30}$ verificaram a correlação entre o MS e os PE das vértebras T1, T10, L3, S1 em posição estática e dinâmica, evidenciando intensa perturbação da pele na saída da posição estática para a dinâmica, ocasionando deslocamentos do MS no sentido CC e LL, especialmente na região lombar. Esses achados reforçam o pressuposto levantado pelo presente estudo de que a palpação dos PE deva ocorrer também na posição em que se deseja avaliar o indivíduo, não unicamente na PO, como é usualmente realizado.

Para Harlick et al. ${ }^{24}$, clínicos têm dificuldade em localizar os PE identificados por meio da superfície da pele. Em compensação, Haneline e Young ${ }^{25}$ evidenciaram que vários estudos apresentam boa concordância na identificação dos $\mathrm{PE}$, principalmente na região lombar. Os achados de Haneline e Young ${ }^{25}$ diferem de Harlick et al..$^{24}$ e corroboram em parte com o presente estudo, cujos deslocamentos de MS na PO para a maioria das vértebras avaliadas foram mínimos, podendo ser praticamente excluído o erro palpatório do avaliador.

Embora o presente estudo não tenha encontrado diferenças significativas entre a PO e DLD, em ambos os eixos apenas para as vértebras T12 e L4, cabe comentar que os valores médios de deslocamento do MS nas duas posições, especialmente para a vértebra L4, apresentaram variabilidade superior a $20 \mathrm{~mm}$, superando a média de $12 \mathrm{~mm}$, encontrada por Downey et al. ${ }^{23}$.

Sob o ponto de vista anatômico, os PE das vértebras da região toracolombar estão localizados e são palpáveis sobre a linha mediana posterior que se localiza no fundo do sulco mediano do dorso, tendo as vértebras T1 até T10 características estruturais semelhantes ${ }^{35}$. Entretanto, T11 e T12 são vértebras torácicas atípicas, as quais podem ser mais difíceis a identificação, sendo utilizados pontos anatômicos de referência que facilitam a identificação desses processos espinhosos ${ }^{21}$. Levando em conta esta dificuldade, é razoável supor que tenham ocorrido, no presente estudo, maiores erros de palpação da vértebra T12 na PO, comparativamente às demais vértebras. Considerando ainda os elevados desvios padrão, obtidos nos deslocamentos em DLD, provavelmente devido à maior espessura de tecido mole encontrado nessa região ${ }^{35}$, especula-se que estas questões poderiam explicar a falta de diferenças significativas nesta região. 
Não existe na literatura uma padronização em relação às técnicas de palpação das proeminências ósseas, assim como não há evidências de um padrão-ouro no método de palpação espinhal, devido à variabilidade da espécie humana ${ }^{31}$. Entretanto, uma boa base científica, assim como a habilidade do fisioterapeuta em identificar as saliências ósseas, indica qualidade das medidas utilizadas em procedimentos de avaliação postural ${ }^{4}$. Desse modo, para que o fisioterapeuta possa planejar e implementar um tratamento corretivo postural, é importante ter conhecimento anatômico e morfológico do sistema musculoesquelético, assim como a compreensão e a aplicação de uma metodologia coerente ao segmento que deseja avaliar.

\section{CONCLUSÃO}

A posição dos MS, identificados por palpação na $\mathrm{PO}$, foi alterada com a modificação da postura para o DLD, ou seja, a localização do processo espinhoso identificado pelo método palpatório, utilizando os MS, não foi condizente com os processos espinhosos identificados no exame radiológico na maioria das vértebras, quando comparadas as PO e DLD. Desta forma, é de extrema importância que a aplicação de uma metodologia de avaliação postural seja coerente à situação que se deseja avaliar.

\section{REFERÊNCIAS}

1. Verderi E. Programa de educação postural. São Paulo: Phorte Editora; 2001.

2. Castro PCG, Lopes JAF. Avaliação computadorizada por fotografia digital como recurso de avaliação na Reeducação Postural Global. Acta Fisiátrica. 2003;10(2):83-88.

3. Sacco ICN, Melo MCS, Rojas GB, Naki I, Burgi K, Silveira LTY, et al. Análise biomecânica e cinesiológica de posturas mediante fotografia digital: estudos de casos. Rev Bras Cien e Mov. 2003;11(2):25-33.

4. Sacco ICN, Alibert S, Queiroz BWC, Pripas D, Kieling I, Kimura AA, et al. Confiabilidade da fotogrametria em relação a goniometria para avaliação postural de membros inferiores. Rev Bras Fisioter. 2007;11(5):411-7.

5. Iunes DH, Castro FA, Salgado HS, Moura IC, Oliveira AS, Bevilaqua-Grossi D. Confiabilidade intra e interexaminadores e repetibilidade da avaliação postural pela fotogrametria. Rev Bras Fisioter. 2005;9(3):327-34.

6. Leroux MA, Zabjek K, Simard G, Badeaux J, Coillard $\mathrm{C}$, Rivard $\mathrm{CH}$. A noninvasive anthropometric technique for measuring kyphosis and lordosis: an application for idiopathic scoliosis. Spine. 2000;25(13):1689-94.

7. Willner S. Spinal pantograph: a non-invasive technique for describing kyphosis and lordosis in the thoracolumbar spine. Acta Orthopaedica Scandinavica. 1981;52:525-9.

8. Charrière L, Roy J. Kinésithérapie des deviations antéropostérieures du rachis et de l'épiphysite vertébrale. Paris: Masson; 1975.

9. Bryant JT, Reid JG, Smith BL, Stevenson JM. Method for determining vertebral body positions in the sagittal plane using skin markers. Spine. 1989;14(3):258-65.

10. Baraúna MA, Canto RST, Sanchez HM, Bustamente JCF, Ventura-Silva RA, Malusá S. Validade e confiabilidade intra-indivíduo do cifolordômetro na avaliação da convexidade torácica. Rev Bras Fisioter. 2005;9(3):319-25.

11. Teixeira FA, Carvalho GA. Confiabilidade e validade das medidas da cifose torácica através do método flexicurva. Rev Bras Fisioter. 2007;11(3):199-204.

12. Öhlén G, Spangfort E, Tingvall C. Measurement of spinal sagittal configuration and mobility with Debrunner's kyphometer. Spine. 1989:580-3.

13. Mellin G. Measurement of thoracolumbar posture and mobilitywith a Myrin inclinometer. Spine. 1986;11:75962.

14. D'osualdo F, Schierano S, Iannis M. Validation of clinical measurement of kyphosis with a simple instrument, the arcometer. Spine. 1997;22:408-22.

15. Souza FR, Ferreira F, Narciso FV, Makhoul CMB, Canto RST, Baraúna MA. Avaliação da concavidade lombar pelo método radiográfico e pela cifolordometria. Rev Bras Fisioter. 2009;13(2).

16. Mercadante FA, Okai LA, Duarte M. Avaliação postural quantitativa através de imagens bidimensionais. In: IX Congresso Brasileiro de Biomecânica, João Pessoa, 2005. Anais. João Pessoa, 2005.

17. Pereira OS. A utilização da análise computadorizada como método de avaliação das alterações posturais: um estudo preliminar. Fis Mov. 2003;16(2):17-25.

18. Silva FC. Avaliação de um programa computacional para a medida da lordose lombar. 2005. 61f. Dissertação (Mestrado em Medicina: Epidemiologia) - Faculdade de Medicina, Universidade Federal do Rio Grande do Sul, Porto Alegre; 2005.

19. Singer KP, Jones TJ, Breidahl PD. A comparison of radiographic and computer-assisted measurements of thoracic and thoracolumbar sagital curvature. Skeletal Radiology. 1990;19:21-6.

20. Portal do projeto software para avaliação postural. São Paulo: Incubadora Virtual Fapesp; 2004. Disponível em: 


\section{Referências (cont.)}

$<$ http://sapo.incubadora.fapesp.br/portal >. Acessado em: 8 de junho de 2010.

21. Normand MC, Descarreaux M, Harrison DD, Harrison DE, Perron DL, Ferrantelli JR, et al. Three dimensional evaluation of posture in standing with the PosturePrint: an intra- and inter-examiner reliability study. Chiropractic \& Ostheopaty. 2007;15(15).

22. Huysmans T, Haex B, De Wilde T, Van Audekercke R, Vander Sloten J, Van Der Perre G. A 3D active shape model for the evaluation of the alignment of the spine during sleeping. Gait Post. 2006;4:54-61.

23. Downey BJ, Taylor NF, Niere KR. Manipulative physiotherapists can reliably palpate nominated lumbar spinal levels. Manual Therapy. 1999;4(3):151-6.

24. Harlick JC, Milosavljevic S, Milburn PD. Palpation identification of spinous processes in the lumbar spine. Manual Therapy. 2007;12:56-62.

25. Haneline MT, Young M. A review of intraexaminer and interexaminer reliability of static spinal palpation: a literature synthesis. Journal of Manipulative and Physiological Therapeutics. 2009;32(5):379-86.

26. Sampaio RF, Mancini MC, Gonçalves GGP, Bittencourt NFN, Miranda AD, Fonseca ST. Aplicação da classificação internacional de funcionalidade, incapacidade e saúde (cif) na prática clínica do fisioterapeuta. Rev Bras Fisioter. 2005;9(2):129-36.

27. Brenzikofer R, Barros RML, Lima Filho EC, Toma E, Bordini LS. Alterações no dorso e coluna vertebral durante marcha. Rev Bras Biomec. 2000;1(1):21-6.

28. Saad M, Masiero D, Lourenço AF, Battistella LR. Proposta de um método de avaliação quantitativa da postura deitada baseado em fotografia. Acta Fisiátrica. 2004;11(2):60-6.

29. Engsberg JR, Lenke LG, Bridwell KH, Uhrich ML, Trout CM. Relationships between spinal landmarks and skin surface markers. J App Biomechan. 2008;24:94-7.

30. Degenhardt BF, Snider KT, Snider EJ, Johnson JC. Interobserver Reliability of Osteopathic Palpatory Diagnostic Tests of the Lumbar Spine: Improvements From Consensus Training. JAOA. 2005;105(10):465-73.

31. Billis EV, Foster NE, Wright CC. Reproducibility and repeatability: errors of three groups of physiotherapists inlocating spinal levels by palpation. Manual Therapy. 2003;8(4):223-32.

32. Moga PJ. Skin distraction at select landmarks on the spine midline in the upright and fully flexed postures. Journal of Bodywork and Movement Therapies. 2009;1-6.

33. Mörl F, Blickhan R. Three dimensional relation of skin markers to lumbar vertebrae of healthy subjects in different postures measured by open MRI. Eur Spine J. 2006;15:742-51.

34. Oriá RB, Ferreira FVA, Santana EN, Fernandes MR, Brito GAC. Estudo das alterações relacionadas com a idade na pele humana, utilizando métodos de histo-morfometria e autofluorescência. An Bras Dermatol. 2003;78(4):425-34.

35. Moore K. Anatomia orientada para clínica. 3 ed. Rio de Janeiro: Guanabara Koogan; 1994.

36. Neumann D. Cinesiologia do aparelho musculoesquelético: fundamentos para reabilitação física. Rio de Janeiro: Guanabara Koogan; 2006.

37. Piva JP, Garcia PCR, Santana JCB, Barreto SSM. Insuficiência respiratória na criança. J Ped. 1998:74(1). 